\title{
Multiple Episodes of Full-Body Tremors: An Unexpected Adverse Effect during an Alzheimer's Disease Investigational Drug Study
}

\section{Jeffrey T Apter, Ivy A Billones* and Kaylee White}

Princeton Medical Institute, Princeton, USA

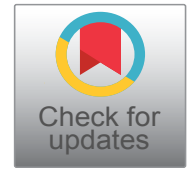

*Corresponding author: Ivy A Billones, Princeton Medical Institute, 256 Bunn Dr \#6, Princeton, NJ 08540, USA

\section{Introduction}

Full-body tremors was not a known or a documented adverse effect $(A E)$ in an investigational drug for prodromal Alzheimer's disease (AD) that reduces beta-amyloid plaques, proteins that may be involved in causing AD.

\section{Case Description}

A 69-year-old Caucasian female experienced multiple episodes of full-body tremors during the extension phase of the clinical study. The investigational drug was taken in the mornings. The patient reported that the AE started six months prior with episodes occurring 1-2 times per week eventually escalating almost daily and was more apparent during midnight hours and upon waking up. Further review of progress notes states that the patient denied the $A E$ and even stated full compliance and no complaints as per protocol before admitting to three missed doses later on.

Patient and trial partner were informed accordingly and in return provided consents acknowledging the risks, the benefits and their roles throughout the entire duration of the study after satisfying the eligibility study criteria. Concomitant medical review, clinical and cognitive assessment scales as well as urinalysis, blood sampling, dermatological exam, monitoring for dementia progression and +/- brain imaging were conducted during her clinical study screening visit and the subsequent clinical study visits. Patient and trial partner were encouraged to report any $A E$, discomfort, missed doses or any concerns.

Her medical history reveals gradual memory decline, most notably forgetfulness of words and conversations for the past three years. Patient's Mini-Mental Status Examination (MMSE) is 24; she is fully oriented; handles problems with moderate difficulty; experiences mild but definite impairment functioning at home; and requires assistance to function independently in community affairs. She denies history of strokes, seizures and family history of memory difficulties.

Patient admits to a previously failed prescription AD drug for 6 months followed by a participation in a separate investigational drug study for memory loss more than two years before commencing the current study. Any ongoing medications including over-the-counter medications, vitamin and herbal supplements were reviewed to have no interactions with the study drug or to suspect to cause serious adverse effects before granting admission to the study.

Immediately right after the AE report, a full physical exam and neurological exam was performed by a study doctor. Results were all within normal range. There was no loss of consciousness (LOC), confusion, bladder incontinence, rigidity of the limbs, vertigo, abnormal gait or cranial nerve (CN) functions, motor and sensory abnormalities, headaches, seizures or residual tremors. Most recent Magnetic Resonance Imaging (MRI) report mentions stable cerebral atrophy and age-related changes, consistent with other MRI reports in comparison to the previous years.

The case of full-body tremors is a case of unknown etiology. Patient was referred to a neurologist for further consultation and shortly thereafter, she opted for an early termination from further participation and cessation of the study drug. A follow-up phone call a few

Citation: Apter JT, Billones IA, White K (2018) Multiple Episodes of Full-Body Tremors: An Unexpected Adverse Effect during an Alzheimer's Disease Investigational Drug Study. Int J Neurodegener Dis 1:004.

Accepted: November 14, 2018; Published: November 16, 2018

Copyright: (C) 2018 Apter JT, et al. This is an open-access article distributed under the terms of the Creative Commons Attribution License, which permits unrestricted use, distribution, and reproduction in any medium, provided the original author and source are credited. 
weeks later confirmed no further AE episodes or other complaints. The study doctor's final report states no reason to suspect progression to any form of dementia.

\section{Discussion}

Acknowledging full-body tremors as an $A E$ has its implications: the $A E$ may or may not be related to the study drug; the AE may arise due to missed doses; and, delayed reporting or missed reporting of an AE may im- pede proper follow-up of care and may otherwise have been prevented from happening in larger clinical trials.

\section{Trial Registration No}

$$
\text { NCT01953601. }
$$

\section{Competing Interests}

The author(s) declare(s) that they have no competing interests. 